\title{
PRINCIPAIS ALTERAÇÕES BUCAIS EM IDOSOS E A IMPORTÂNCIA DA ABORDAGEM MULTIPROFISSIONAL
}

\author{
MAIN CHANGES IN ELDERLY PEOPLE AND THE IMPORTANCE OF \\ THE MULTIPROFESSIONAL APPROACH
}

\author{
Adyelle Dantas Ribeiro ${ }^{1}$ \\ Layrlla Kateriny Moura Oliveira Lopes ${ }^{2}$ \\ Waleska Fernanda Souto Nóbrega ${ }^{3}$
}

RESUMO: OBJETIVO: Revisar a literatura acerca da importância da equipe multidisciplinar no cuidado e atenção à saúde bucal do idoso. MÉTODO: Foram realizadas buscas bibliográficas nas seguintes bases de dados: Lilacs, Medline, Bireme, SciELO e PubMed, utilizando os seguintes descritores: "Assistência Integral à Saúde do Idoso", "Odontologia Geriátrica", "Manifestações Bucais", e dos respectivos termos em inglês: "Comprehensive Health Care", "Geriatric Dentistry" e "Oral Manifestations". Foram incluídos artigos científicos publicados entre 2010 e 2020. Foram excluídas as teses, dissertações e estudos que não abordavam a temática principal da presente pesquisa. RESULTADOS: Verificou-se que os idosos fazem parte de um grupo populacional que apresenta, frequentemente, alterações fisiológicas e patológicas diversas, tanto relacionadas à saúde geral como à saúde bucal, podendo estar associadas ao uso crônico de medicamentos, aos hábitos de alimentação e higiene e à própria motivação do paciente. CONCLUSÃO: É necessária uma abordagem multidisciplinar em todas as fases de atendimento do idoso, desde o diagnóstico precoce, assim como durante o planejamento e tratamento integrado, para contribuir na melhoria de sua qualidade de vida.

Palavras chave: Assistência integral à saúde. Manifestações bucais. Odontologia geriátrica.

ABSTRACT: OBJECTIVE: To review the literature on the importance of the multidisciplinary team in the care and attention to the oral health of the elderly.

\footnotetext{
${ }_{1}$ Doutoranda em Clínicas Odontológicas, Mestre em Saúde e Sociedade, Universidade Estadual da Paraíba - UEPB, Campina Grande - PB.

2 Doutoranda em Clínicas Odontológicas, Mestre em Saúde e Sociedade, Universidade Estadual da Paraíba - UEPB, Campina Grande - PB.

3 Doutoranda em Clínicas Odontológicas, Mestre em Saúde Pública, Universidade Estadual da Paraíba - UEPB, Campina Grande - PB.
} 
METHOD: Bibliographic searches were performed in the following databases: Lilacs, Medline, Bireme, SciELO and PubMed, using the following descriptors: "Comprehensive Health Care for the Elderly", "Geriatric Dentistry", "Oral Manifestations", and the respective terms in English: "Comprehensive Health Care", "Geriatric Dentistry" and "Oral Manifestations". Scientific articles published between 2010 and 2020 were included. Theses, dissertations and studies that did not address the main theme of this research were excluded. RESULTS: It was found that the elderly are part of a population group that frequently presents different physiological and pathological changes, both related to general health and oral health, which may be associated with chronic use of medications, eating habits and hygiene and the patient's own motivation. CONCLUSION: A multidisciplinary approach is required in all phases of care for the elderly, from early diagnosis, as well as during planning and integrated treatment, to contribute to improving their quality of life.

Keywords: Comprehensive health care. Oral manifestations. Geriatric dentistry. 


\section{INTRODUÇÃO}

No Brasil, o segmento populacional que mais cresce é o de idosos. Segundo os dados de projeção do Instituto Brasileiro de Geografia e Estatística, a população de idosos deverá atingir 41,5 milhões em 2030 (MIRANDA, LIA \& MIRANDA 2010). Esse rápido envelhecimento torna fundamental ao profissional de saúde, conhecer a realidade da população onde atua, devendo o cirurgião-dentista dominar as condições de saúde bucal para assim conseguir identificar os problemas e planejar ações que favoreçam esse grupo específico.

De acordo com Ribeiro e colaboradores (2012), observa-se na Odontologia, uma clientela idosa em busca de tratamentos voltados as suas prioridades. Alterações fisiológicas decorrentes do envelhecimento da cavidade bucal podem ocasionar desconforto e insatisfação nos idosos. A conscientização e valorização dos cuidados especiais por parte dos cirurgiões-dentistas devem ser observadas diante da necessidade do paciente, assim como, o reconhecimento da saúde bucal como indispensável a uma boa qualidade de vida do idoso.

O aumento da expectativa de vida populacional nem sempre se faz acompanhado de saúde, já que processo de envelhecimento provoca várias alterações no organismo. Geralmente, idosos usam maior número de medicamentos, o que pode ocasionar alterações sistêmicas e promover efeitos adversos na cavidade oral. Estudos apontam que as patologias bucais presentes em idosos podem ser justificadas pelas diferentes mudanças que envolvem esses pacientes como um todo, sendo elas: o próprio processo de envelhecimento, mudanças metabólicas, fatores nutricionais, uso de medicamentos, uso de próteses, hábitos psicopatológicos, uso de álcool e uso de tabaco (MEIRA, et al., 2018).

Sendo assim, o presente artigo tem como objetivo realizar uma revisão de literatura sobre as principais alterações bucais decorrentes do envelhecimento, ressaltando a importância de uma equipe multidisciplinar no cuidado e atenção à saúde bucal do idoso. 


\section{MATERIAIS E MÉTODO}

Foi realizada uma revisão crítica da literatura por meio da busca bibliográfica nas bases de dados Lilacs, Medline, Bireme, SciELO e PubMed. As publicações indexadas nessas bases de dados foram identificadas por meio dos seguintes descritores: "Assistência Integral à Saúde do Idoso", "Odontologia Geriátrica", "Reabilitação Bucal", e dos respectivos termos em inglês: "Comprehensive Health Care", "Geriatric Dentistry" e "Mouth Reabilitation". Inicialmente, os estudos foram selecionados apenas pelos títulos e resumos. Foram incluídos artigos científicos publicados entre 2010 e 2020. Foram excluídas as teses, dissertações e estudos que não abordavam a temática principal da presente pesquisa.

\section{REVISÃO DE LITERATURA}

Segundo Silva e colaboradores (2011), a saúde sistêmica do idoso deve ser cautelosamente analisada pelo odontólogo, já que algumas condições decorrentes do envelhecimento podem surgir na cavidade bucal, como: redução da capacidade gustativa, alterações nas glândulas salivares / xerostomia e alterações no periodonto. A equipe odontológica deve tratar a saúde de modo multi e interdisciplinar, agindo a favor do entendimento da pessoa idosa, suas particularidades e entendendo a fundo seu contexto biopsicossocial.

Nas Diretrizes da Política Nacional de Saúde Bucal, propostas pelo Ministério da Saúde (MS), é ressaltada a importância da saúde bucal dos idosos como fator decisivo para a manutenção de uma boa qualidade de vida. O acesso ao atendimento odontológico é essencial para manter a boa saúde bucal e, aliado aos cuidados médicos, deve estar voltado para a prevenção de doenças e melhora da qualidade de vida do idoso (DUTRA \& SANCHEZ, 2015). 
Conforme Cardoso \& Lago 2010, a saúde bucal não se separa da saúde geral; fatores ambientais e gerais do indivíduo impactam o sistema estomatognático e vice-versa, o que torna a compreensão dessas relações muito relevantes para o diagnóstico das verdadeiras prioridades e necessidades do idoso, assim como para planejamento de ações. Deste modo, o trabalho com a terceira idade requer a composição de uma vasta rede de conhecimentos de mão dupla.

A abordagem dos pacientes idosos precisa ser distinta daquela voltada à população como um todo, já que o envelhecimento promove alterações fisiológicas que inclinam o idoso a possuir, frequentemente, patologias próprias do envelhecimento, o que demanda cuidado por parte dos profissionais de saúde. $\mathrm{O}$ discernimento destas alterações na pessoa idosa possibilita ao cirurgião-dentista um atendimento direcionado e específico, o que acarreta qualidade e eficácia clínica (CÔRTE-REAL, FIGUEIRAL \& CAMPOS, 2011).

\section{Impacto Na Qualidade De Vida}

Diante do exposto por Domingos, Moratelli \& Oliveira em 2011, pesquisas sobre a influência das condições bucais na qualidade de vida do idoso mostram que as questões funcionais, emocionais e sociais são expressivamente influenciadas por uma condição bucal deficiente. Isto torna-se ainda mais preocupante quando se reflete que grande parte dos idosos não possui acesso ao tratamento odontológico necessário, o que constantemente ocorre por despreparo profissional em enxergar essa condição como uma necessidade de atuação interdisciplinar.

\section{Saúde Bucal Do Idoso}

A saúde é fruto do equilíbrio por inteiro que é o organismo, dessa maneira, a saúde bucal é parte indissociável da saúde geral. Assim, uma vez prejudicada, 
perturba a saúde geral, prejudicando a nutrição, a fala e o bem-estar físico e social do idoso. Desse modo, as perdas dentárias são provenientes de uma saúde bucal precária, associada aos fatores sociodemográficos como raça, baixa renda, tabagismo, gênero e baixa escolaridade (ARAÚJO, 2012).

\section{Uso De Medicamentos}

De acordo com Gontijo e colaboradores (2012), os idosos representam o maior grupo de usuários de medicamentos do mundo, variando entre antidepressivos, anti-hipertensivos, descongestionantes, diuréticos, antiácidos, antirreumáticos, antiarrítmicos cardíacos, anticolinérgicos, laxantes e imunossupressores. Essas medicações podem influenciar no tratamento odontológico, devendo o cirurgião-dentista dialogar com o médico geriatra, a fim de analisar a administração desses fármacos, manter boas condições de saúde bucal e orientar os idosos, seus cuidadores e familiares sobre uma adequada higiene oral, evitando repercussões negativas na saúde geral (MEIRA, et al., 2018).

Além disso, segundo Paula e colaboradores (2014), algumas doenças sistêmicas e o próprio uso de medicamentos podem causar efeitos negativos na secreção salivar, levando a um aumento do risco para o aparecimento de lesões bucais na população idosa, como estomatites, candidíase, periodontites. Como consequência, estes pacientes necessitam de um acompanhamento odontológico para avaliar, prevenir e tratar essas alterações bucais associadas ao uso de medicamentos sistêmicos (SILVA, et al. 2017).

\section{Condições Neurológicas}

Conforme afirmado por Meira e colaboradores (2018), o envelhecer propicia mudanças fisiológicas e patológicas, bem como variações no aspecto psicossocial, 
que podem facilitar o aparecimento de alterações bucais. Além disso, essas modificações podem agir em juntamente com as doenças bucais, refletindo no grau de autonomia, independência e qualidade de vida do idoso. O estado de saúde bucal dos idosos é influenciado ainda pela presença de doenças neurológicas, visto que essas patologias afetam os cuidados pessoais, e isso reflete na higiene bucal, que se torna prejudicada.

A demência que mais afeta os idosos é a doença de Alzheimer, um desarranjo mental de origem neurodegenerativa que afeta o Sistema Nervoso Central (SNC) e culmina na perda gradual das habilidades cognitivas, advinda da morte de células cerebrais. Os idosos com este quadro tornam-se progressivamente mais incapacitados de realizar atividades diárias básicas, como a higiene bucal, repercutindo na vida social. As especificidades de cada etapa do Alzheimer interferem diretamente nas prioridades do paciente e devem ser consideradas em um adequado plano de tratamento odontológico (MIRANDA, LIA \& MIRANDA, 2010).

Segundo Bulgareli e colaboradores (2018), o mal de Parkinson é outra doença neurológica, crônica e progressiva, que consiste na degradação de neurônios do SNC atuantes no controle dos movimentos, ocasionando tremor, rigidez, movimentação lenta, alteração do equilíbrio e diversas dificuldades ligadas às atividades diárias, inclusive a escovação dentária.

\section{Alterações Orais}

As estruturas bucais sofrem ação do tempo, e diversas são as alterações fisiológicas que ocorrem no ciclo da dentição normal. A dentina depositada constantemente reduz o tamanho da câmara pulpar, causando a atresia dos condutos radiculares. Os tecidos periodontais sofrem retração, perda do colágeno e tornam-se mais suscetíveis a inflamação. As mudanças mastigatórias são inúmeras, advindas da perda dos elementos dentários, uso de próteses, restaurações realizadas no decorrer da vida e hábitos alimentares e funcionais (DUTRA \& 
SANCHEZ, 2015). Ademais, algumas condições têm sua prevalência elevada em decorrência da fisiologia do envelhecimento, como será abordado em sequência.

\section{Cárie Dentária}

De acordo com Fernandes-Costa e colaboradores (2013), dados globais a cárie é a doença bucal mais prevalente entre os indivíduos com sessenta anos ou mais. Fatores como a queda do fluxo salivar proveniente do uso de certas medicações, dificuldade motora na higienização e as mudanças na dieta potencializam diretamente os efeitos dessa doença. As lesões cariosas podem evoluir levando à degradação dos tecidos periodontais, predispondo o indivíduo ao surgimento de cáries radiculares (ALBENY, 2018).

\section{Xerostomia}

Segundo Lucena e colaboradores (2010), O principal sintoma dessa condição é a sensação de boca seca, podendo ser originada por alterações nas glândulas salivares, que sofem um processo de degeneração avançada ou do uso constante de medicações, podendo estar associada ao uso de anti-hipertensivos e digitálicos. Outros medicamentos relacionados à queda do fluxo salivar são os analgésicos, anticonvulsivantes, anticolinérgicos, antieméticos, anti-histamínicos, descongestionantes, diuréticos e psicotrópicos.

Essas alterações degenerativas também afetam a viscosidade da saliva secretada, especialmente em repouso. Ademais, os pacientes podem apresentar redução na produção da amilase salivar, tendo a digestão de carboidratos na cavidade bucal prejudicada e, consequentemente, a deglutição e posterior digestão do bolo alimentar dificultadas. (MEIRA, 2018). 


\section{Candidose}

Conforme estudo de Paula e colaboradores (2014), uma das lesões mais comum na população idosa é a candidose, em virtude do ambiente favorável para a colonização e proliferação de Candida spp. A Candidose Pseudomembranosa aguda é uma das variações clínicas mais comuns de manifestação da doença, que afeta principalmente indivíduos imunossuprimidos ou acometido por doenças crônicas. Expõe-se, clinicamente, como placas ou nódulos branco-amarelados que são facilmente removidas com gazes ou cotonete.

A Candidose Eritematosa é uma lesão sintomática, em consequência das numerosas erosões distribuídas pela mucosa e à inflamação presente, causando dor intensa, e ocorre principalmente no dorso da língua. Em sua forma crônica, as lesões são assintomáticas, provocando apenas ardência durante a ingestão de alimentos ácidos ou quentes, apresentando algumas variantes clínicas que acometem, frequentemente, pacientes idosos, como a Atrófica Crônica e a Queilite Angular (SIQUEIRA, 2014).

Diante do exposto por Simões e colaboradores (2013), a Candidose Atrófica Crônica consiste em alterações inflamatórias presentes abaixo da prótese total maxilar, podendo atingir até $65 \%$ dos idosos que fazem uso de prótese, principalmente a prótese total superior e pode ocorrer em menor frequência na mucosa de suporte da prótese parcial removível. Dentre seus fatores etiológicos, sobressaem-se o traumatismo crônico, próteses mal adaptadas, a não remoção da prótese durante a noite e a má higiene do paciente.

Em idosos que apresentam perda de dimensão vertical de oclusão, é comum o acúmulo de saliva nas comissuras labiais, propiciando a proliferação de Candida spp, causando a queilite angular. Alguns medicamentos como atrovastatina, busulfan, clofazimina, metildopa, ritonavir, sais de ouro, sinvastatina, tetraciclina, indinavir e vitamina $A$ também têm sido associados à presença desta lesão, visto que favorecem o acúmulo de saliva na comissura labial, e facilitam o crescimento de 
microorganismos principalmente a Candida albicans na cavidade bucal, além de reduzir a resposta imunológica (PAULA, ALMEIDA \& ALVES, 2014).

\section{Hiperplasia Gengival Medicamentosa}

Segundo estudo de Fernandes e colaboradores (2013), caracteriza-se pelo aumento do volume gengival provocado pelo uso de medicações que atuam junto ao metabolismo dos fibroblastos e do colágeno. Diversos medicamentos, incluindo o ácido tranexâmico, anlodipina, cetoconazol, ciclosporina, cotrimoxazol, eritromicina, felodipina, fenitoína, nicardipina, verapamil, vigabratina, nifedipina e pirimidona têm sido relacionados ao sobrecrescimento gengival.

A presença de hiperplasia gengival induzida por fármacos em pacientes desdentados não é comum e, quando acontece, está associada a fatores irritantes locais como próteses e a deposição de placa bacteriana sobre elas, já em pacientes dentados, geralmente inicia-se entre o primeiro e o terceiro mês da terapia com a medicação, aumentando apenas nos próximos doze a dezoito meses (MENDES, CERQUEIRA \& AZOUBEL, 2014).

\section{Condição Periodontal}

De acordo com Meira et al. (2018), o processo de envelhecimento também é refletido nos tecidos periodontais, cujo nível de comprometimento aumenta com a idade, levando à uma queda na cicatrização e a um acelerado avanço da doença periodontal. Essas alterações periodontais são marcadas por: irregularidades na superfície do cemento e do osso alveolar, alteração quantitativa de tecido mineralizado na cortical e no trabeculado ósseo, elevação da reabsorção e redução da formação óssea. 


\section{Edentulismo}

Se bem cuidados, os dentes naturais podem permanecer durante toda a vida do indivíduo, portanto o edentulismo não é uma consequência natural do envelhecimento. As perdas dentárias ocasionam mudanças físicas, biológicas e emocionais. Dentre estas alterações nota-se a função mastigatória deficiente, levando a escolha por alimentos mais fáceis de mastigar, que geralmente são ricos em carboidratos e gorduras, favorecendo o surgimento de cárie, doença periodontal e posteriormente a perda de outros elementos dentários (OLIVEIRA, DELGADO \& BRESCOVICI, 2014).

Segundo Agostinho, Campos \& Silveira (2015), a perda dentária interfere na fonética, vivenciada através da dificuldade ou da impossibilidade de pronunciar corretamente as palavras, deixando o sujeito com comunicação e expressão desfavoráveis. Reflete também na insatisfação estética, visto que desencadeia estímulos negativos na autoestima impactando diretamente as relações sociais e afetivas, já que a ausência dos dentes acentua as linhas de envelhecimento e as rugas, alterando o rosto.

O indivíduo pode ainda estar sujeito à rejeição social e ao preconceito, e, provavelmente, esta inquietação o faz ter dificuldade em assumir-se desdentado. E acabam, muitas vezes, reagindo de forma conformista e depressiva, enfrentando a perda dos dentes como algo próprio à idade, mostrando-se passivos diante da situação (ARAÚJO, et al., 2012).

\section{DISCUSSÃO}

A crescente população de idosos torna necessária uma atenção especial para o tratamento odontológico desses indivíduos. Araújo et al. (2012), revisando a 
literatura, abordaram o emergente envelhecimento da população mundial, inclusive no Brasil, destacando a necessidade de medidas para lidar com esta situação.

A condição de saúde bucal da população idosa é menosprezada, mesmo sendo parte indissociável da saúde geral dos indivíduos, percebendo-se através dos baixos índices de procura ao serviço odontológico. Nota-se que os próprios pacientes idosos, seus familiares, cuidadores e mesmo os demais profissionais não enxergam a relevância de uma boa saúde bucal e a necessidade de tratamento odontológico, mesmo com a falta ou ausência total de elementos dentários (ROCHA \& MIRANDA, 2013).

De acordo com Catão, Xavier \& Pinto (2011), o avançar da idade carrega também alterações na cavidade oral destes pacientes, sendo a maioria delas consequência do estado geral de saúde, tais como efeito colateral de alguns medicamentos, dificuldade motora na realização da higiene bucal, e acesso pouco frequente aos cuidados preventivos.

É papel do Cirurgião-Dentista proporcionar ao paciente idoso um tratamento diferenciado, sugerindo meios de prevenção dessas condições bucais, além de analisar melhores formas de conduzir o tratamento e orientá-los sobre a importância da higienização bucal, independentemente da quantidade de elementos dentários presentes em boca (ALBENY \& SANTOS, 2018).

Segundo Meira et al. (2018), deve-se observar a necessidade de encaminhar o idoso ao médico para que este ateste as suas condições de saúde e autorize a realização do tratamento e/ou do procedimento cirúrgico. Assim sendo, é evidente a importância do cirurgião-dentista em uma equipe multidisciplinar para acompanhamento e tratamento de pacientes idosos.

O envelhecer é um resultado natural do desenvolvimento compreendido por uma soma de modificações morfológicas e fisiológicas. As modificações sofridas no sistema estomatognático dos pacientes geriátricos possuem grande frequência clínica e requerem conhecimentos aprofundados na literatura, proporcionando informações que colaborem para o aprimoramento desta área odontológica (ALBENY \& SANTOS, 2018).

Por isso, conforme expôs Ciminino \& Reis (2014), a equipe de saúde bucal deve estar inserida numa abordagem de saúde com caráter multi e interdisciplinar; 
atuando a favor do entendimento do idoso, suas particularidades, conhecendo seu contexto biopsicossocial, a fim de que o plano de tratamento vise a saúde do indivíduo na sua integralidade.

\section{CONCLUSÕES}

Foi apresentado o papel do odontólogo na esfera da pessoa idosa, bem como as principais particularidades desses indivíduos, no que diz respeito às condições bucais e às considerações sistêmicas. Além disso, destacou-se a importância do atendimento multiprofissional com o desenvolvimento interdisciplinar na contribuição do atendimento e acompanhamento da pessoa idosa à luz da integralidade em saúde.

\section{REFERÊNCIAS BIBLIOGRÁFICAS}

AGOSTINHO, A.C.M.G.; CAMPOS, M.L.; SILVEIRA, J.L.G.C; Edentulismo, uso de prótese e autopercepção de saúde bucal entre idosos; Rev Odontol UNESP. Mar.-Apr.; 44(2): 74-79; 2015.

ALBENY, A.L.; SANTOS, D.B.F; Doenças Bucais que mais acometem o paciente na terceira idade: Uma revisão de literatura; Id on Line Rev. Mult. Psic. V.12, N. 42, p. 681-694, ISSN 1981-1179; 2018.

ARAÚJO, I.S.T.; FREITAS, I.N.; SILVA, R.B.; VASCONCELOS, M.G.; VASCONCELOS, R.G.; Odontologia e abordagem interdisciplinar na atenção integral ao idoso relacionado às principais alterações orais; Com. Ciências Saúde.; 23(1):1-102; 2012.

BULGARELI, J.V.; FARIA, E.T.; CORTELLAZZI, K.L.; GUERRA, L.M.; MENEGHIM, M.C.; AMBROSANO, G.M.B.; FRIAS, A.C.; PEREIRA, A.C.; Fatores que influenciam o impacto da saúde bucal nas atividades diárias de adolescentes, adultos e idosos; Rev Saude Publica.;52:44; 2018.

CARDOSO, M.B.R.; LAGO, E.C.; Alterações bucais em idosos de um centro de convivência; Revista Paraense de Medicina V.24 (2) abril-junho 2010.

CATÃO MHCV, XAVIER AFC, PINTO TCA. O impacto das alterações do sistema estomatognático na nutrição do idoso. Rev Bras de Ciên da Saúd. 29(9):73-8; 2011.

CIMINO, A.M.T.; REIS, J.R.; Avaliação da Saúde Bucal do Idoso em uma instituição de apoio a idosos no Distrito Federal; Com. Ciências Saúde. 25(3/4): 237-244; 2014. 
CÔRTE-REAL, I.S.; FIGUEIRAL, M.H.; CAMPOS, J.C.R.; As doenc,as orais no idoso Considerações gerais; Rev Port Estomatol Med Dent Cir Maxilofac. 52(3):175-180; 2011.

DOMINGOS, P.A.S.; MORATELLI, R.C.; OLIVEIRA, A.L.B.M.; Atenção Odontológica Integral ao Idoso: Uma Abordagem Holística; Revista de Odontologia da Universidade Cidade de São Paulo. 23(2): 143-53, mai-ago; 2011.

DUTRA, C.E.S.V.; SANCHEZ, H.F.; Organização da atenção à saúde bucal prestada ao idoso nas equipes de saúde bucal da Estratégia Saúde da Família; Rev. Bras. Geriatr. Gerontol., 18(1):179-188; Rio de Janeiro; 2015.

FERNANDES-COSTA AN, VASCONCELOS MG, QUEIROZ LMG, BARBOZA CAG, VASCONCELOS RG. As Principais Modificações Orais que ocorrem durante o Envelhecimento. Rev Brasileira de Ciências da Saúde. 17(3): 293-300; 2013.

GONTIJO, M.F.; RIBEIRO, A.Q.; KLEIN, G.H.; ROZENFELD, S.; ACURCIO, F.A.; Uso de antihipertensivos e antidiabéticos por idosos: inquérito em Belo Horizonte, Minas Gerais, Brasil; Cad. Saúde Pública, Rio de Janeiro, 28(7):1337-1346, jul, 2012.

MEIRA, I.A.; MARTINS, M.L.; MAICEL, P.P.; CAVALCANTI, Y.W.; ARAÚJO, T.P.; PIAGGE, C.S.L.D.; Multidisciplinaridade no cuidado e atenção à saúde bucal do idoso; Rev. Ciênc. Méd.;27(1):39-45; 2018.

MENDES, T.E.B.; CERQUEIRA, L.B.; AZOUBEL, M.C.F.; Aumento gengival influenciado por drogas; Revista Bahiana de Odontologia. Jan;5(1):29-37; 2014.

MIRANDA, F.A.; LIA, E.N.; LEAL, S.C.; MIRANDA, M. P.A. F.; Doença de Alzheimer: características e orientações em odontologia; RGO, Porto Alegre, v. 58, n.1, p. 103-107, jan./mar. 2010.

OLIVEIRA, B.S.; DELGADO, S.E.; BRESCOVICI, S.M.; Alterações das funções de mastigação e deglutição no processo de alimentação de idosos institucionalizados; Rev. Bras. Geriatr. Gerontol., Rio de Janeiro; 17(3):575- 587; 2014.

PAULA, B.G.; ALMEIDA, M.R.B.; ALVES, J.F.C.S.; Alterações Bucais De Idosos Institucionalizados - Revisão De Literatura; Rev. Odontol. Univ. Cid. São Paulo; 26(3): 219-26, set-dez; 2014.

RIBEIRO, A.F.L.; LEAL, M.C.C.; MARQUES, A.P.O.; Importance of geriatric dentistry to elderly nutrition; RGO - Rev Gaúcha Odontol., Porto Alegre, v.60, n.2, p. 241-246, abr./jun., 2012.

ROCHA, D.A.; MIRANDA, A.F.; Atendimento odontológico domiciliar aos idosos: uma necessidade na saúde bucal nas atividades diárias de adolescentes, adultos e idosos; Rev Saude Publica;52:44; 2018.

SILVA, E.M.M.; BARÃO, V.A.R.; SANTOS, D.M.; DELBEN, J.A.; RIBEIRO, A.C.P.; GALLO, A.K.G; Principais alterações e doenças bucais que acometem o paciente geriátrico - revisão da literatura; Odonto; 19 (37): 39-47; 2011.

SILVA, H.P.R.; KOPPE B.; BREW, M.C.; SÓRIA, G.S.; BAVARESCO, C.S.; Abordagem das afecções bucais mais prevalentes em idosos: uma revisão integrativa com foco na atenção primária; Rev. Bras. Geriatr. Gerontol., Rio de Janeiro; 20(3): 432-443; 2017. 